\title{
ANÁLISE DO PERFIL CLÍNICO DE PACIENTES COM HANSENÍASE RECIDIVANTE DO MUNICÍPIO DE SOBRAL
}

\author{
* Lorena Alves Trajano I \\ Miguel Marcelo Freire de Melo \\ José Francisco Igor Siqueira Ferreira '
}

\begin{abstract}
RESUMO
A hanseníase é uma doença de forte impacto na saúde pública brasileira, tanto pela dificuldade no diagnóstico, por parte dos profissionais de saúde, como pela possibilidade de recidiva anos após a apresentação inicial da doença. Este estudo teve como objetivo avaliar o perfil epidemiológico e clínico dos pacientes com casos de hanseníase no município de Sobral-CE, entre o período de 2012 a 2018, com análise especial para os casos de recidiva da doença. Os dados foram obtidos por meio do banco de dados do Sistema de Informação de Agravos de Notificação (SINAN) do município. Foram evidenciados 42 casos de hanseníase recidivante no período estudado, sendo mais da metade destes ocorridos em pessoas com faixa etária entre 41 e 70 anos de idade. Houve maior predominância de reincidência em pessoas do sexo feminino, da raça parda e com menor nível de escolaridade. A grande parte das recidivas foram classificadas como multibacilar e as formas clínicas predominantes foram dimorfa e virchowiana. Conclui-se que o perfil epidemiológico foi composto, em sua maioria, por pacientes de faixa etária mais avançada, raça parda e com baixa escolaridade. O perfil clínico predominante de doentes na recidiva foi com a forma multibacilar. Tais dados foram congruentes com os obtidos na literatura. Além disso, existe uma importante parcela de dados que não foi notificada corretamente no SINAN, dificultando a confiabilidade das informações.
\end{abstract}

PALAVRAS-CHAVE: Saúde Pública. Epidemiologia. Infectologia.

Acadêmica do curso de Medicina. Universidade Federal do Ceará, UFC - Campus Sobral. Departamento de Medicina. CEP: 62042280, Sobral, Ceará, Brasil. *Autor correspondente: Ioren.tj21@gmail.com ORCID: 0000-0002-9591-7617; 0000-0002-3744-3273; 0000-0001-9905-1577 


\section{INTRODUÇÃO}

\begin{abstract}
A hanseníase é uma doença infecciosa causada pelo Mycobacteriumleprae(M.leprae). É considerada potencialmente incapacitante e, embora curável, seu diagnóstico causa grande impacto psicossocial, pelos preconceitos e estigmas que a envolvem. ${ }^{1}$ É considerado caso de hanseníase o portador de um ou mais dos seguintes sintomas: lesão de pele com alteração de sensibilidade; acometimento de nervo com espessamento neural e baciloscopia positiva. ${ }^{2}$
\end{abstract} A doença é classificada em quatro formas definidas - indeterminada, tuberculoide, dimorfa e virchowiana - , segundo o grau de resposta imunológica. ${ }^{3}$

Em 2017, o Brasil foi o primeiro responsável pela endemia no continente sulamericano e pelo segundo maior número absoluto de casos da doença no mundo. ${ }^{4}$ Neste mesmo ano, 210.671 novos casos foram detectados no mundo. 5 Antes da década de 1940, a hanseníase era considerada uma doença intratável. Na década de 1980, a Organização Mundial de Saúde (OMS) recomendou a dapsona, a rifampicina e a clofazimina como agentes primários para a terapia multidrogas. ${ }^{6}$ Segundo a classificação operacional, são utilizadas associações farmacológicas distintas: doentes paucibacilares (PBs) recebem por seis meses dapsona diariamente e rifampicina, administração mensal supervisionada; multibacilares (MBs) são tratados durante doze meses com dapsona e clofazimina diariamente e doses mensais supervisionadas de rifampicina e clofazimina. ${ }^{7}$

Casos de recidiva em hanseníase são raros em pacientes tratados regularmente. Usualmente, recidivas ocorrem em um período maior que cinco anos após a cura. ${ }^{8}$ Há importantes diferenças de registros de recidivas em regiões brasileiras nas quais a prevalência da doença é alta, como na Amazônia.,9,10 De acordo com o Ministério da Saúde, os critérios para diagnóstico da recidiva da doença $\mathrm{PB}$ são: paciente que, após diagnóstico de cura, apresentar dor nas raízes nervosas, com novas lesões ou exacerbações de lesões prévias que não respondem ao tratamento com corticoides, por pelo menos de 90 dias; e pacientes com surto reacional tardio, geralmente cinco anos após ser considerado curado. ${ }^{8}$

Para os casos MBs: pacientes que, após o tratamento completo e cura, apresentam lesões cutâneas e/ou exacerbação de lesões antigas, novas alterações neurológicas que não respondem ao tratamento com talidomida e/ou corticoides nas doses e esquemas recomendados; baciloscopia positiva e clínica compatível em pacientes não tratados anteriormente; e pacientes com surto reacional tardio, geralmente cinco anos após ser considerado curado. ${ }^{8}$ Quando um paciente inicialmente $\mathrm{PB}$ recidiva como $\mathrm{MB}$, isso provavelmente significa que houve um erro de classificação durante o tratamento inicial. Outro fator importante é o IB (Índice Baciloscópico) alto no início e no fim do tratamento, quando a recidiva ocorre com mais facilidade. ${ }^{11}$

A adesão a terapia varia ampla e globalmente: $<70 \%$ na hanseníase MB e $90 \%$ na PB. ${ }^{12} A$ irregularidade do tratamento pode determinar a ocorrência de recidivas, em função do aparecimento de resistência medicamentosa. ${ }^{13}$ As recidivas constituem o principal indicador da efetividade de um programa de controle da hanseníase. Tornamse necessários estudos que abordem as variáveis envolvidas na ocorrência de recidivas, para auxiliar na implementação e avaliação dos programas e na melhoria dos resultados no tratamento da hanseníase. ${ }^{11}$ 
O objetivo desse trabalho é avaliar o perfil epidemiológico e clínico dos pacientes com casos de hanseníase no município de

\section{MATERIAL E MÉTODOS}

Trata-se de um estudo de abordagem quantitativa, epidemiológico, transversal, descritivo, observacional e individuado dos pacientes que passaram por tratamento poliquimioterápico para hanseníase, atendidos pelo Sistema Único de Saúde (SUS) no município de Sobral, localizado na Região Norte do estado do Ceará, no período compreendido entre 2012 e 2018.

Os dados foram obtidos através da análise das Fichas de Notificação/Investigação do banco de dados do Sistema de Informação de Agravos de Notificação (SINAN), na Vigilância Epidemiológica da Secretaria de Saúde do município de Sobral.

Para o perfil pessoal, considerou-se: unidade da Estratégia Saúde da Família ao qual o paciente estava vinculado, sexo, idade, raça e escolaridade. Quanto ao perfil dos casos, incluíram-se: número de lesões cutâneas, forma clínica, classificação operacional, baciloscopia, esquema terapêutico inicial, duração do tratamento e tipo de saída. Os indivíduos incluídos no estudo foram aqueles que receberam tratamento para hanseníase e que, posteriormente, apresentaram recidiva da doença, perfazendo um total de 42 pacientes assim distribuídos nos últimos 7 anos: 6 recidivas em 2012, 10 recidivas em 2013, 6 recidivas em
Sobral-CE, entre o período de 2012 a 2018, com análise especial para os casos de recidiva da doença.

2014, 8 recidivas em 2015, 4 recidivas em 2016, 5 recidivas em 2017 e 3 recidivas em 2018. As variáveis analisadas baseiam-se tanto no perfil pessoal do paciente quanto no perfil geral dos casos. Foram excluídos do estudo todos os pacientes que não apresentaram recidiva nos últimos 7 anos.

Os dados foram processados em uma planilha do Software Microsoft Excel e em seguida convertidos para o formato de banco de dados utilizando o Software IBM SPSS (Statistical Package For The Social Science Versão 23 para Windows). A análise descritiva e exploratória dos dados foi efetuada inicialmente por meio das distribuições de frequências.

O projeto foi apresentado à Comissão Científica da Secretaria de Saúde do município de Sobral e ao Comitê de Ética em Pesquisa (CEP) da Universidade Estadual Vale do Acaraú (UVA), sendo aprovado pelo número do parecer (Certificado de Apresentação para Apreciação Ética - CAAE) 2.261.007.

Esse estudo beneficia a comunidade acadêmica ao servir de parâmetro para futuras pesquisas a respeito do tema. Poucos estudos na literatura analisam pacientes com hanseníase que fizeram ou fazem tratamento e que recidivaram.

\section{RESULTADOS E DISCUSSÃO}

Quanto à idade no que se refere ao diagnóstico, observa-se baixo percentual de casos de hanseníase em idades iniciais, sendo o primeiro caso, ou seja, o diagnóstico inicial da doença, ocorrido em $2,38 \%$ dos pacientes com 9 a 10 anos e $4,76 \%$ com 11 a 20 , e os casos de 
recidiva em $0 \%$ para pacientes com menos de 10 anos e apenas $4,76 \%$ com idade entre 11 e 20 anos. A faixa etária juvenil, de 20 a30, se mostrou com quantidade intermediária, representada por $11,9 \%$ no primeiro caso e $7,14 \%$ na recidiva. Já as faixas de 40 a 70 anos apresentaram maior número de casos, tanto com diagnóstico inicial como de recidiva, representadas no primeiro diagnóstico por $16,6 \%$ de 41 a $50,23,8 \%$ de 51 a 60 e $14,28 \%$ de 61 a 70 anos, e na recidiva por $28,57 \%, 23,8 \%$ e $21,4 \%$, respectivamente. Por fim, quanto à faixa correspondente às idades de 71 a 80 anos, teve representatividade apenas nos casos de recidiva, sendo observada em 7,14\% dos casos (Tabela 1).

TABELA 1: Idade dos pacientes no diagnóstico inicial de hanseníase e na recidiva no município de Sobral-CE, 2012 a 2018

\begin{tabular}{ccccc}
\hline Idade (anos) & $\begin{array}{c}\text { Primeiro Caso } \\
\left(\mathbf{n}^{\circ} \text { de casos) }\right.\end{array}$ & Primeiro Caso $(\%)$ & $\begin{array}{c}\text { Recidiva } \\
\left(\mathbf{n}^{\circ} \text { de casos }\right)\end{array}$ & $\begin{array}{c}\text { Recidiva } \\
(\%)\end{array}$ \\
\hline 9 a 10 & 1 & 2,38 & 0 & 0 \\
11 a 20 & 2 & 4,76 & 2 & 4,76 \\
21 a 30 & 5 & 11,90 & 3 & 7,14 \\
31 a 40 & 11 & 26,20 & 3 & 7,14 \\
41 a 50 & 7 & 16,60 & 12 & 28,57 \\
51 a 60 & 10 & 23,80 & 10 & 23,80 \\
61 a 70 & 6 & 14,28 & 9 & 21,40 \\
71 a 80 & 0 & 0 & 3 & 7,14 \\
\hline \hline
\end{tabular}

Como observado, o maior número de casos de recidiva corresponderam a pacientes com faixa etária entre 41 e 70 anos, sendo esses dados condizentes com a literatura, que relata idade como fator de risco para a doença recidivante. Casos multibacilares são mais frequentes nas faixas etárias mais avançadas, enquanto casos paucibacilares entre os mais jovens. ${ }^{14}$

Em relação ao sexo dos pacientes nesse estudo, $22(52,38 \%)$ dos 42 casos eram do sexo feminino, enquanto 20 (47,61\%) eram do sexo masculino, sem diferença numérica importante entre ambos. Apesar de, neste estudo, pouco mais de $50 \%$ dos casos corresponderem ao sexo feminino, já foi demonstrado em outras pesquisas que as desigualdades de gênero apresentam forte influência no processo saúde-doençacuidado, sendo que os homens se tornam mais vulneráveis ao adoecimentoe a formas mais graves, não apenas pela forma de viver a sua masculinidade, mas também à inadequação dos serviços de saúde na identificação e atendimento de suas necessidades específicas de saúde. ${ }^{15}$ Foi relatado que o sexo masculino é o mais atingido; nas mulheres, as recidivas mantêm relação com a gravidez e a lactação. ${ }^{14}$

O Ministério da Saúde, em agosto de 2016, publicou nota técnica informativa de alertava para o exame sistemático de hanseníase na população masculina e em idosos. Esse documento foi baseado na análise de 541.090 casos novos de hanseníase notificados no período de 2001-2013, cujos resultados mostraram chances duas 
vezes maiores para a ocorrência de casos multibacilares nestas duas populações. Fatores biológicos, hormonais e imunossenescência também são discutidos e reforçam esse maior risco. ${ }^{16}$

No que diz respeito a raça, números e frequências dessa estão descritos na Tabela 2. Pode-se notar predomínio da raça parda, representada por $47,61 \%$ dos casos. Quanto às demais, em ordem crescente, houve representação de $7,14 \%$ de brancos e $21,42 \%$ de pretos. $23,8 \%$ dos casos não tiveram sua raça registrada no prontuário, sendo classificada como ignorada.

Em relação à escolaridade dos indivíduos, nota-se um percentual baixo em pacientes com níveis de escolaridade mais elevados, sendo representados por apenas $2,38 \%$ com ensino superior completo, $0 \%$ com ensino superior incompleto bem como ensino médio completo e 11,9\% com ensino médio incompleto. Em relação às demais categorias, que correspondem a maioria dentre os estudados, $19 \%$ eram de analfabetos, $45,23 \%$ com ensino fundamental incompleto e $4,76 \%$ com fundamental completo. Além disso, dentre os 42 casos, $16,6 \%$ não tiveram sua escolaridade registrada no prontuário, classificadas, desta forma, como ignorada (Tabela 2).

TABELA 2: Distribuição dos pacientes com hanseníase recidivante por raça e escolaridade no município de Sobral-CE, 2012 a 2018

\begin{tabular}{ccc}
\hline Raça & Número de pacientes & Porcentagem dos casos (\%) \\
\hline Branca & 3 & 7,14 \\
Parda & 20 & 47,61 \\
Preta & 9 & 21,42 \\
Ignorada & 10 & 23,80 \\
\hline Escolaridade & & \\
\hline Analfabeto & 8 & 19,00 \\
Fundamental Incompleto & 19 & 45,23 \\
Fundamental Completo & 2 & 4,76 \\
Ensino Médio Incompleto & 5 & 11,90 \\
Ensino Médio completo & 0 & 0 \\
Ensino Superior Incompleto & 0 & 0 \\
Ensino Superior completo & 1 & 2,38 \\
Ignorado & 7 & 16,60 \\
\hline
\end{tabular}

Fonte: Sinan/Ministério da Saúde/Secretaria de Vigilância em Saúde

Portanto, é notável que a pouca escolaridade está presente na maioria dos pacientes estudados, podendo estar relacionada ao baixo nível de informação e, desse modo, à baixa adesão medicamentosa ou à terapêutica inadequada, fatores cruciais para a não ocorrência de recidivas.

Em relação ao número de lesões cutâneas apresentadas, 33,3\% dos pacientes não apresentaram nenhuma lesão cutânea no momento do diagnóstico inicial, enquanto, quando houve recidiva, apenas $1(2,38 \%)$ caso 
não apresentou lesão. Do restante da amostra, 9 (21,42\%) pacientes apresentaram lesão única no primeiro diagnóstico e $6(14,28 \%)$ na recidiva. Quanto aos casos que apresentaram duas a cinco lesões, houve uma proporção igual para primeiro caso e recidiva: 11 (26,2\%) pacientes. Em pacientes que apresentaram mais de 5 lesões cutâneas houve uma diferença significativa do número de casos do primeiro diagnóstico e da recidiva, sendo identificados 8 (19\%) no primeiro caso e $18(42,85 \%)$ na recidiva. Além disso, em seis prontuários dos casos de recidiva não havia informações sobre a quantidade de lesões (Tabela 3).

TABELA 3: Distribuição dos pacientes portadores de hanseníase durante o diagnóstico inicial e a recidiva por número de lesões, forma clínica, classificação operacional e resultado da baciloscopia no município de SobralCE, 2012 a 2018

\begin{tabular}{|c|c|c|c|c|}
\hline Número de lesões & $\begin{array}{c}\text { Primeiro Caso } \\
\left(\mathrm{n}^{\circ} \text { de casos }\right)\end{array}$ & Primeiro Caso (\%) & $\begin{array}{c}\text { Recidiva } \\
\left(\mathrm{n}^{\circ} \text { de casos }\right) \\
\end{array}$ & $\begin{array}{c}\text { Recidiva } \\
(\%)\end{array}$ \\
\hline 0 & 14 & 33,30 & 1 & 2,38 \\
\hline 1 & 9 & 21,42 & 6 & 14,28 \\
\hline $2-5$ & 11 & 26,20 & 11 & 26,20 \\
\hline$>5$ & 8 & 19,00 & 18 & 42,85 \\
\hline Não informado & 0 & 0 & 6 & 14,28 \\
\hline \multicolumn{5}{|l|}{ Forma Clínica } \\
\hline Indeterminada & 9 & 21,42 & 9 & 21,40 \\
\hline Tuberculoide & 10 & 23,80 & 3 & 2,14 \\
\hline Dimorfa & 17 & 40,47 & 9 & 21,40 \\
\hline Virchowiana & 6 & 14,28 & 3 & 2,14 \\
\hline Não classificada & 0 & 0 & 9 & 21,40 \\
\hline \multicolumn{5}{|l|}{$\begin{array}{l}\text { Classificação } \\
\text { Operacional } \\
\end{array}$} \\
\hline Paucibacilar & 17 & 40,47 & 4 & 9,50 \\
\hline Multibacilar & 25 & 59,52 & 38 & 90,47 \\
\hline \multicolumn{5}{|l|}{ Baciloscopia } \\
\hline Positiva & 4 & 9,50 & 16 & 38,10 \\
\hline Negativa & 7 & 16,60 & 13 & 30,90 \\
\hline Não Realizada & 3 & 7,14 & 9 & 21,42 \\
\hline Ignorada & 28 & 66,60 & 4 & 9,50 \\
\hline
\end{tabular}

Fonte: Sinan/Ministério da Saúde/Secretaria de Vigilância em Saúde

Observa-se que houve tendência de as recidivas se apresentarem com maior quantidade de lesões cutâneas em comparação com o primeiro diagnóstico e, portanto, com uma mudança no que diz respeito a classificação operacional. Essa classificação é baseada na 
alocação de pacientes com menos de seis lesões de pele para o grupo $\mathrm{PB}$ e pacientes com seis ou mais lesões para o grupo MB. A confiabilidade da classificação apenas nesses critérios tem sido questionada, pois um sistema baseado na contagem de lesões é propenso a subestimar o número de lesões e categorizar erroneamente pacientes com menos de seis lesões como PBs e, portanto, subestimar a gravidade clínica de parte dos casos. ${ }^{17}$

Considerando a forma clínica da hanseníase, observa-se que as manifestações clínicas da forma indeterminada estiveram presentes em $9(21,42 \%)$ pacientes ao primeiro diagnóstico e em apenas $3(7,14 \%)$ pacientes que recidivaram. A forma tuberculoide foi detectada em $10(23,8 \%)$ pacientes no primeiro caso e em $2(4,76 \%)$ recidivantes. Já a forma dimorfa foi encontrada em $17 \quad(40,47 \%)$ pacientes ao primeiro diagnóstico e em 19 $(45,23 \%)$ que recidivaram. Quanto à forma virchowiana, um quadro mais grave da doença, detectou-se $6(14,28 \%)$ pacientes no primeiro diagnóstico e 14 (33,3\%) na recidiva. Por fim, nenhum paciente deixou de ser classificado ao primeiro diagnóstico, entretanto, 4 (9,5\%) deles não foram classificados quando recidivaram (Tabela 3).

No que diz respeito à classificação operacional dos pacientes analisados, no primeiro diagnóstico, 17 (40,47\%) foram definidos como PBs, enquanto 25 (59,52\%) foram identificados como MBs. Já nos casos de recidivas, 4 (9,5\%) eram PBs e 38 (90,47\%) MBs. A classificação operacional do doente é importante por definir o tempo e o esquema de tratamento (Tabela 3).

Observa-se, portanto, que os casos de recidiva foram em sua maioria diagnosticados como MBs, tendo provavelmente uma parte destes recebido o diagnóstico e, consequentemente, o tratamento, de forma errada no primeiro caso.

O risco de recaída está associado tanto à sensibilidade quanto ao valor preditivo de um teste, ou método para classificar corretamente um MB (VPP). Segundo Bührer-Sékula et al, a chance de categorizar corretamente um paciente MB foi de $71,3 \%$ (VPP). ${ }^{17}$

Um estudo enfatizou que, embora não houvesse muita resistência do bacilo individual contra dapsona ou rifampicina, uma parcela substancial dos casos tratados de hanseníase multibacilar ainda não estava mostrando resposta microbiológica com evidência de redução genômica, sugerindo que esse grupo possa precisar de uma atenção especial, pois pode haver uma tendência crescente de evasão da defesa do hospedeiro, o que pode levar a futura persistência da doença até mesmo à sua recaída. ${ }^{16}$

Quanto à baciloscopia, percebese que, no diagnóstico inicial, 4 (9,5\%) dos pacientes possuíam baciloscopia positiva, 7 $(16,6 \%)$ negativa, em $3(7,14 \%)$ destes, o exame não foi realizado, e, em 28 (66,6\%) casos essa informação foi ignorada. Já nos casos de recidiva, a maioria, $16(38,1 \%)$ casos, foi positiva, seguida por 13 (30,95\%) negativos, $9(21,42 \%)$ não realizados e 4 (9,5\%) ignorados (Tabela 3 ).

Apesar de discreta, a diferença entre o primeiro caso e a recidiva no que tange à baciloscopia, no estudo de Bührer-Sékula et al. ${ }^{17}$ é demonstrado que, com base no risco de recidiva após a Terapia Multidrogas (MDT), aconselha-se atenção especial aos pacientes com hanseníase com alta carga bacteriana e à baciloscopia.

Noquetangeaoesquemadetratamento dos pacientes com primeiro diagnóstico, dos 42 casos, 14 (33,3\%) completaram o tratamento com seis doses mensais supervisionadas de rifampicina e dapsona, por um período de até 9 meses; 21 (50\%) utilizaram doze doses mensais supervisionadas de rifampcina, dapsona e clofazimina em até 18 meses e 7 $(16,6 \%)$ foram tratados com outros esquemas. Sobre os 42 pacientes que tiverem recidivas, 
4 (9,5\%) foram tratados com seis doses e 38 (90,47\%) com doze doses (Tabela 4).

Embora o programa de eliminação da hanseníase tenha tido grande sucesso na Índia e em outros países, a taxa anual de detecção de novos casos de hanseníase continua sendo motivo de preocupação. Estudos relataram o problema da persistência da bactéria em casos tratados de hanseníase, mesmo após a conclusão da terapia multidrogas (MDT). Além disso, foi relatado que o efeito ambiental sobre o genoma bacteriano também pode fazê-lo agir contra a atividade das drogas, daí a possibilidade de recaída, reinfecção e resistência, mesmo após a instituição de uma terapêutica adequada conforme as orientações atuais. ${ }^{16}$

Sobre o tempo de duração do tratamento, descrito na Tabela 4, ao primeiro diagnóstico dos pacientes com hanseníase $M B$, $16 \%$ realizaram até 12 meses de tratamento, $72 \%$ entre 12 a 18 meses e os $12 \%$ restantes tiveram o tempo de tratamento maior que 18 meses. Em relação à duração do tratamento dos pacientes com hanseníase PB ao primeiro diagnóstico, $17,64 \%$ fizeram o tratamento em até 6 meses, $52,95 \%$ fizeram em 6 a 9 meses e $29,41 \%$ fez em 9 meses ou mais. No que diz respeito aos casos da recidiva dos pacientes com hanseníase $M B$, $18,42 \%$ tiveram o tempo de tratamento de até 12 meses e $65,78 \%$ fizeram o tratamento em 12 a 18 meses, não havendo nenhum registro de tratamento em 18 meses ou mais. Os pacientes restantes $(15,8 \%)$ tiveram seu tempo de tratamento ignorado no preenchimento da ficha de notificação.

Já os pacientes recidivantes, com diagnóstico de hanseníase PB, $25 \%$ fizeram o tratamento em até 6 meses, 75\% tiveram seu tratamento com duração de 6 a 9 meses, e nenhum teve tratamento com 9 meses ou mais de duração. Observamos nesse caso que a maioria realizou a terapêutica no período determinado de acordo com a classificação.

TABELA 4: Agrupamento dos pacientes portadores de hanseníase quanto ao esquema e a duração do tratamento realizado durante o diagnóstico inicial e durante a recidiva no município de Sobral - CE, 2012 a 2018

\begin{tabular}{|c|c|c|c|c|c|c|c|c|}
\hline $\begin{array}{l}\text { Esquema de Tratamento } \\
\text { (Doses supervisionadas) }\end{array}$ & \multicolumn{2}{|c|}{$\begin{array}{c}\text { Primeiro Caso } \\
\text { (n }{ }^{\circ} \text { de casos) }\end{array}$} & \multicolumn{2}{|c|}{ Primeiro Caso (\%) } & \multicolumn{2}{|c|}{$\begin{array}{c}\text { Recidiva } \\
\left(\mathrm{n}^{\circ} \text { de casos }\right)\end{array}$} & \multicolumn{2}{|c|}{$\begin{array}{c}\text { Recidiva } \\
(\%)\end{array}$} \\
\hline Seis doses & \multicolumn{2}{|l|}{14} & \multicolumn{2}{|l|}{33} & \multicolumn{2}{|l|}{4} & \multicolumn{2}{|l|}{9,5} \\
\hline Doze doses & \multicolumn{2}{|l|}{21} & \multicolumn{2}{|l|}{50} & \multicolumn{2}{|l|}{38} & \multicolumn{2}{|c|}{90,47} \\
\hline Outros esquemas & \multicolumn{2}{|l|}{7} & \multicolumn{2}{|l|}{17} & \multicolumn{2}{|l|}{0} & \multicolumn{2}{|l|}{0} \\
\hline \multirow{2}{*}{$\begin{array}{c}\text { Duração do Tratamento } \\
\text { (meses) }\end{array}$} & \multicolumn{2}{|c|}{ Primeiro Caso PB } & \multicolumn{2}{|c|}{ Primeiro Caso PB } & \multicolumn{2}{|c|}{ Primeiro Caso PB } & \multicolumn{2}{|c|}{ Primeiro Caso PB } \\
\hline & $\mathrm{N}^{\mathrm{a}}$ de casos & $\%$ & $\mathrm{~N}^{\mathrm{a}}$ de casos & $\%$ & $\mathrm{~N}^{\mathrm{a}}$ de casos & $\%$ & $\mathrm{~N}^{\mathrm{a}}$ de casos & $\%$ \\
\hline$\leq 6$ & 3 & 17,64 & - & - & 3 & 25 & - & - \\
\hline 6 a 9 & 9 & 52,95 & - & - & 9 & 75 & - & - \\
\hline$\geq 9$ & 5 & 28,41 & - & - & 5 & 0 & - & - \\
\hline$\leq 12$ & - & - & 4 & 16 & - & - & 7 & 18,42 \\
\hline 12 a 18 & - & - & 18 & 72 & - & - & 25 & 65,78 \\
\hline$\geq 18$ & - & - & 3 & 12 & - & - & 0 & 0 \\
\hline Ignorado & 0 & - & 0 & 0 & 0 & 0 & 6 & 15,80 \\
\hline
\end{tabular}


O estudo da OMS sobre risco de recaída da hanseníase julga desnecessário acompanhar e examinar cuidadosamente os pacientes após a MDT. ${ }^{20}$ Isso se baseia na observação de que a MDT é eficiente e que a maioria dos estudos de acompanhamento mostra apenas uma baixa porcentagem de recaídas. No entanto, ao acompanhar pacientes com altas cargas bacterianas, antes do tratamento por períodos mais longos, a taxa de recaída aumenta 20 vezes. $^{18}$

Por fim, a respeito do tipo de saída, no primeiro diagnóstico, todos os 42 pacientes foram classificados como curados. Já nos casos

\section{CONCLUSÕES}

Conclui-se, portanto, que o perfil avaliado dos diagnósticos de hanseníase redicivante consiste em pacientes em faixas etárias mais avançadas, especialmente entre 41 e 70 anos.

A raça parda teve predomínio dentre os casos estudados, representando $47,61 \%$ e, no que diz respeito à escolaridade, pacientes com escolaridade mais baixa, em especial os analfabetos e aqueles com ensino fundamental incompleto, apresentaram maior representatividade, 19 e $45,23 \%$ respectivamente.

Em relação ao perfil clínico, observouse primeiramente que, no que se refere ao número de lesões, o primeiro diagnóstico não demonstrou diferença apreciável dentre os participantes. Entretanto, na recidiva, 42,85\% dos casos apresentavam mais de 5 lesões cutâneas, expressando, como discutido, mudança na classificação operacional desses pacientes no segundo diagnóstico, sendo $90,47 \%$ dos casos recidivados classificados como Multibacilar. No primeiro diagnóstico, de recidiva, do total, $30(71,4 \%)$ pacientes com hanseníase finalizaram o tratamento como curados, os outros 12 (28,58\%) tiveram a forma de saída ignorada no preenchimento da ficha.

Os dados obtidos são consistentes, em sua maioria, aos dados presentes na literatura no que diz respeito a maior taxa de recidiva em pacientes com maior faixa etária, bem como maior número de diagnósticos na recidiva de casos que se apresentam com mais lesões cutâneas, revelando a possibilidade de erro no primeiro diagnóstico, fato que contribui para a ocorrência da recidiva da doença.

a diferença entre os casos $\mathrm{PB}$ e $\mathrm{MB}$ não era significativa. Além disso, a forma Dimorfa e a Virchowiana foram as predominantes nos casos recidivantes.

No que tange a terapia recomendada, a maior parte dos estudados foi tratado corretamente de acordo com a classificação. Por fim, os 42 pacientes analisados, inicialmente foram classificados como curados quanto ao tipo de saída, no segundo diagnóstico, essa classificação foi representada por $71,4 \%$, sendo o restante não inserido na ficha de acompanhamento.

O presente estudo aponta, também, para a falha no que tange a obtenção de dados epidemiológicos, considerando que nos casos redicivantes, $14,28 \%$ dos pacientes não tiveram número de lesões quantificados, sendo classificados como não informado. De modo geral, em Sobral, muitos aspectos foram catalogados apenas como ignorados, não informado ou não rotulado, prejudicando a obtenção e avaliação mais consistente das informações obtidas. 


\title{
ANALYSIS OF THE CLINICAL PROFILE OF PATIENTS WITH LEPROSY RELAPSE OF THE MUNICIPALITY OF SOBRAL
}

\section{ABSTRACT}

Leprosy is a disease with a strong impact in Brazil's public health, not only because of the difficulty to the diagnosis by the healthcare professionals, but also because the possibility of relapse years after the initial presentation. This study aimed to evaluate the epidemiological and clinical profile of leprosy patients in the municipality of Sobral-CE, between 2012 and 2018, with special analysis for relapsing cases of the disease. The data was obtained through the database of the National Disease Notification System (SINAN) of the municipality. 42 cases of relapsing leprosy were observed during the study period, with more than half of them occurring with people between 41 and 70 years old. Females and mixed race were the most affected. There was also a predominance of cases in people with lower educational level. Most recurrences were classified as multibacillary, and the predominant clinical forms were dimorphic and virchowian. It was concluded that the epidemiological profile was composed mostly by patients of advanced age, mixed race and lower education level. The predominant clinical profile of those with relapsing illness was with the Multi-bacillary form. This data was congruent with the observed in the literature. In addition, there's an important part of the data that wasn't correctly notified in the SINAN's system, complicating the reliability of the information.
\end{abstract}

KEYWORDS: Public Health. Epidemiology. Infectology.

\section{REFERÊNCIAS}

1. Franco MCA, Macedo GMM, Menezes BQ, Jucá Neto FOM, Franco ACA, Xavier MB. Perfil de casos e fatores de risco para hanseníase, em menores de quinze anos, em município hiperendêmico da região norte do Brasil. Rev Paraense Med 2014; 28(4):29-40.

2. Crespo MJI, Gonçalves A, Padovani CR. Hanseníase: pauci e multibacilares estão sendo diferentes. Medicina (Ribeirão Preto) 2014; 47(1):43-50.

3. Gonçalves, A. Realidades do controle da hanseníase: atualizando cenários. Rev Bras Epidemiol 2013; 16:611-21.

4. Global leprosy update, 2017: reducing the disease burden due to leprosy. Weekly Epidemiological 2018; 93, 35: 444-56.
5. Brasil. Ministério da Saúde, Secretaria de Vigilância em Saúde. Boletim Epidemiológico. Caracterização da situação epidemiológica da hanseníase e diferenças por sexo, Brasil, 2012-2016. Bol Epidemiol. 2018; 49.

6. Beltrán-Alzate C, López Días F, Romero-Montoya M, Sakamuri R, Kimura M, Brennan $\mathrm{P}$, Cardona-Castro N. Leprosy Drug Resistance Surveillance in Colombia: The experience of a sentinel country. PLoS Negl Trop Dis. 2016; 10 (10): e0005041.

7. Stefani MMA, Avanzi C, Bührer-Sékula $S$, Benjak A, Loiseau C, Singh $P$, et al. Whole genome sequencing distinguishes between relapse and reinfection in recurrent leprosy cases. PLoS Negl Trop Dis 11(6):e0005598. 
8. Raiol TKA, Volpato SE, Guimarães PB, Lopes MLAA, Takano DM, Santana JM. Atypical lesions in relapsed leprosy. An Bras Dermatol. 2014; 89(5):808-11.

9. Ferreira SMB, Ignotti E, Senigalia LM, Silva DRX, Gamba MA. Recidivas de casos de hanseníase no estado de Mato Grosso. Rev Saude Publica. 2010; 44(4): 650-57.

10. Oliveira MLW. Monitoramento de recidivas e resistência medicamentosa em hanseníase no Brasil, protocolos para investigação clínica e laboratorial. Brasília: Ministério da Saúde; 2010.

11. Bona SH, Campelo V, Holanda AON, Costa UA, Silva LOBV. Recidivas de hanseníase em Centros de Referência de Teresina, Piauí, 2001-2008. Epidemiol Serv Saúde 2015; 24(4):731-38. doi: $10.5123 / \mathrm{S} 1679-49742015000400015$.

12. Kumar A, Girdhar A, Chakma JK, Girdhar BK. WHO Multidrug Therapy for Leprosy: Epidemiology of Default in Treatment in Agra District, Uttar Pradesh, India. BioMed Research International 2015.

13. Büker-Sékula S, Cunha MG, Foss NT, Oskam L, Faber WR, Klatser PR. Dipstick assay to identify leprosy patients who have in increased risk of relapse. Trop Med Int Health. 2001; 6(4):317-23.

14. Kamal S, Thappa DM. Relapse in leprosy. Indian J Dermatol Venereol Leprol. 2009 Mar-Apr;75:126-35.

15. Souza EA, Boigny RN, Ferreira AF, Alencar CH, Oliveira MLW, Ramos Jr. AN. Vulnerabilidade programática no controle da hanseníase: padrões na perspectiva de gênero no Estado da Bahia, Brasil. Cad Saúde Pública 2018; 34:e00196216.

16. Reja AHH, De A, Patra PK, Biswas S, Duttagupta U, Sil A, et al. Genomic Reduction at TTC Repeats in the Bacterial Genome of Treated Cases of Hansen's Disease: A Possible Survival Mechanism of Mycobacterium leprae. Indian J Dermatol. 2018;63(6):449-54. doi: 10.4103/ijd. IJD_90_18.

17. Buhrer-Sekula S, Cunha MG, Foss NT, Oskam L, Faber WR, Klatser PR. Dipstick assay to identify leprosy patients who have an increased risk of relapse. Tropical Medicine and International Health 6: 317-23, 2001.

18. World Health Organization. Leprosy Unit. (1994). Risk of relapse in leprosy. World Health Organization. https://apps.who.int/iris/handle/10665/61868. 